\title{
BioMedicine
}

Volume 10 | Issue 3

Article 6

2020

\section{A Unique Intraluminal growth of Juvenile Nasopharyngeal Angiofibroma: A Case Report}

Follow this and additional works at: https://www.biomedicinej.com/biomedicine

Part of the Life Sciences Commons, and the Medical Sciences Commons (c) (i)

This work is licensed under a Creative Commons Attribution 4.0 License.

\section{Recommended Citation}

Mohammadi Ardehali, Mojtaba; Irani, Shirin; and Firouzifar, Mohammadreza (2020) "A Unique Intraluminal growth of Juvenile Nasopharyngeal Angiofibroma: A Case Report," BioMedicine: Vol. 10 : Iss. 3 , Article 6. DOI: $10.37796 / 2211-8039.1019$

This Case Reports is brought to you for free and open access by BioMedicine. It has been accepted for inclusion in BioMedicine by an authorized editor of BioMedicine. 


\title{
A unique intraluminal growth of juvenile nasopharyngeal angiofibroma: A case report
}

\author{
Mojtaba Mohammadi Ardehali, Shirin Irani*, Mohammadreza Firouzifar
}

Otorhinolaryngology Research Center, Amir Alam Hospital, Tehran University of Medical Sciences, Tehran, Iran

\begin{abstract}
Juvenile nasopharyngeal angiofibroma (JNA) is a rare, hypervascular, benign tumor which is mainly seen among male adolescents. The tumor typically originates from the sphenopalatine fossa, but could spread through natural foramens and fissures.

There are some reports of atypical growth of this tumor in literature but the intraluminal growth, which could be seen in paraganglioma and glomus tumors, has not reported yet in angiofibroma. In this article we present a case of extensive angiofoibroma with intraluminal involvement of the ophthalmic vein.

Our patient was a 19-year-old boy with a complaint of nasal obstruction and occasional epistaxis since a year ago, without any visual or neurologic complaints. The patient underwent an endoscopic resection of the tumor after embolization via the nasal cavity.

The intraoperative findings revealed the tumor extension to the orbit, intracranial space and cavernous sinus via inferior orbital fissure. The intracranial extension of the tumor was extradural and was successfully excised without CSF leakage. An interesting finding in this patient, was an intraluminal extension of the tumor in to the ophthalmic vein, which was completely excised endoscopically. (pre and post operation pictures are available in the full text). The definitive treatment of angiofibroma is surgical excision. Different surgical approaches are used but nowadays endoscopic resection with or without pre-operative embolization is the first choice of treatment. The intraluminal growth of the tumor was also excised as a pedunculated mass separately.
\end{abstract}

Keywords: Angiofibroma, sinus endoscopy, intraluminal growth

\section{Introduction}

$\mathrm{J}$ uvenile nasopharyngeal angiofibroma (JNA) is a rare, hypervascular, benign tumor which is mainly seen among male adolescents [1]. It accounts for $0.05 \%$ of head and neck tumors. Despite its benign histologic pattern, it could have an aggressive clinical trend to invade adjacent anatomic structures [2].

The tumor typically originates from the sphenopalatine fossa, but could spread through natural foramens and fissures. It could involve nasopharynx, the nasal cavity, maxillary and ethmoid sinuses by medial extension. Lateral growth results in pterygopalatine fossa involvement and furtherly extending to infratemporal fossa and cheek via pterygomaxillary fissure.

It could involve the orbit via the inferior orbital fissure from the infratemporal fossa. Also, tumor growth to the parasellar compartment of the middle cranial fossa is seen by means of superior orbital fissure.

Additionally, the encasement of the internal carotid artery and invasion of the cavernous sinus may be seen in advanced intracranial involvement [2].

There are some reports of atypical growth of this tumor in literature but the intraluminal growth, which could be seen in paraganglioma and glomus tumors [3], has not reported yet in angiofibroma. In this article we present a case of extensive angiofoibroma with intraluminal involvement of the ophthalmic vein.

Received 24 December 2019; accepted 3 February 2020

Available online 1 September 2020.

* Corresponding author. Research Center of Otolaryngology and Head \& Neck Surgery, Tehran University of Medical Sciences, Amiralam Hospital, Tehran, Iran. Fax: +982166760269.

E-mail address: sh_irani@razi.tums.ac.ir (S. Irani). 


\section{Case presentation}

A 19-year-old boy referred to our clinic by a complaint of nasal obstruction since a year ago. He complained of progressive obstruction of the right nasal side which extends to bilateral obstruction gradually. He also complained of occasional epistaxis. He did not have any visual or neurologic complaints. The past medical history, family history and drug history was unremarkable.

Rhinoscopic examination, revealed a mass in right nasal cavity which occlude the nose. He had also a mild swelling of the cheek and mild proptosis of the right eye. The visual acuity was detected 10/10 in ophthalmology consultation. Marcus gunn sign was negative and the eye movements were all normal.

The patient has also undergone a pre-operation neurosurgical consultation. The neurological evaluation was unremarkable with normal cranial nerves' function.

The computed tomography scan (CT scan) revealed a large mass in right nasal cavity with destruction of pterygoid plates, the body and the greater wing of the sphenoid, inferior orbital wall and the posterior wall of the maxillary sinus (Fig. 1). There was also evidences of intracranial extension, infratemporal invasion and extension to nasopharynx in the imaging. The mass was so close to right orbital apex. Large vascular structures were noticed in the scan. Also, there was a dilatation of superior ophthalmic vein, facial vein and ethmoidal veins (Fig. 2).

The Magnetic Resonance Imaging (MRI), also endorsed the evidences of CT scan (Fig. 3). A hypervascular mass with plenty of flow voids signal, due to containing large vessels, was detected in the right nasal cavity with extension to intracranial and infratemporal spaces.
The patient was scheduled for endoscopic resection of the tumor, by the first impression of angiofibroma, Radkowski stage of IIIB. Due to its vast extension, the pre-operation embolization was planned 48 hours before surgery. The feeding vessels of the JNA was bilateral internal maxillary arteries and bilateral internal carotid arteries. The bilateral internal maxillary arteries were embolized by PVA 300-350. The intralumininal growth is depicted in the angiographic examination (Fig. 4).

The patient underwent an endoscopic resection of the tumor via the nasal cavity. For optimal access to lateral border of the tumor, a gingivobuccal incision was performed.

The intraoperative findings revealed that the tumor extends to orbit, intracranial space and cavernous sinus via inferior orbital fissure. The intracranial extension of the tumor was extradural and was successfully excised without CSF leakage. An interesting finding in this patient, was an intraluminal extension of the tumor in to ophthalmic vein, which was identified intra-operatively and completely excised endoscopically (Fig. 5). The blood loss volume was $1400 \mathrm{cc}$ and he received 4 pack cell during the endoscopic procedure. The hemoglobin level was $12.7 \mathrm{~g} / \mathrm{dl}$ pre-operatively, while it was $11.7 \mathrm{~g} / \mathrm{dl}$ six hours after blood transfusion. The neurologic examination and visual acuity was intact post operatively. The post-operative period was unremarkable and he was discharged 4 days later. The patient had a recurrence tumor a year later in the lateral portion of the maxilla which was excised endoscopically and did not need other ancillary treatments. The pathology evaluation, confirmed the diagnosis of angiofibroma.

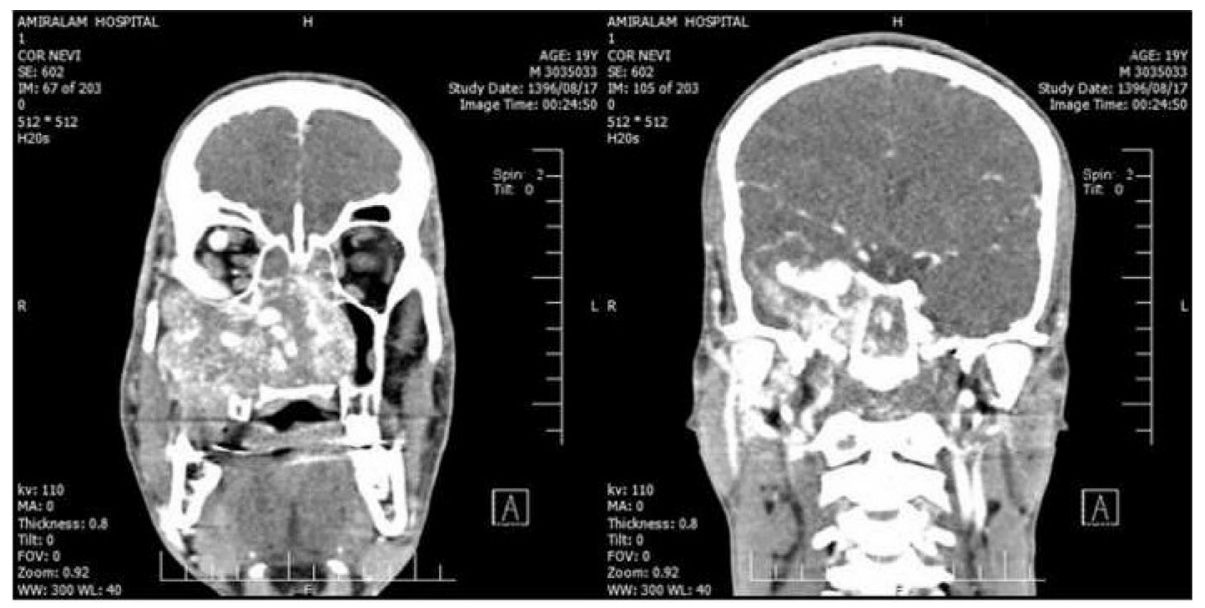

Fig. 1. The computed tomography scan with intravenous contrast revealed a large mass in right nasal cavity with destruction of pterygoid plates, the body and the greater wing of the sphenoid, inferior orbital wall and the posterior wall of the maxillary sinus. 


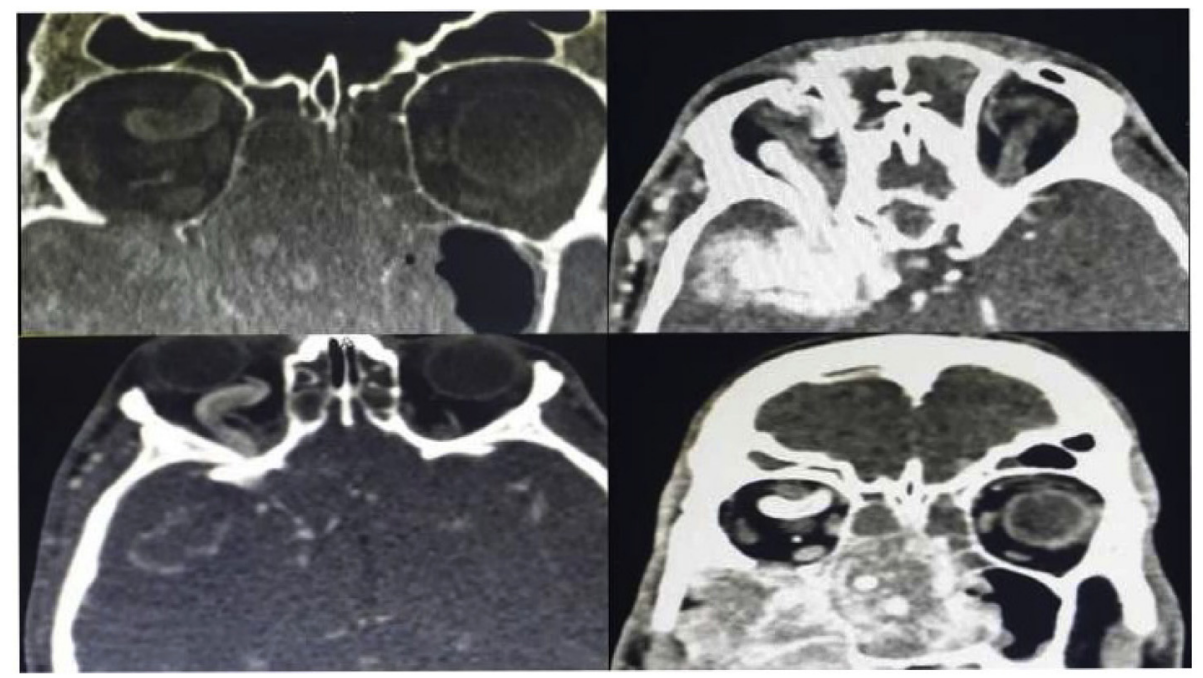

Fig. 2. The computed tomography scan with intravenous contrast revealed a large hypervascular mass in right nasal cavity with orbital involvement.

\section{Discussion}

Juvenile nasopharyngeal angiofibroma is a histologically benign tumor but could have an aggressive behaviour and can invade contiguous structures [4].

The usual pattern of JNA growth is known, but there are some reports about unusual or atypical types of angiofibroma. Morkenborg and his colleagues have presented a case of bilateral angiofibroma which were completely removed by embolization followed by endoscopic surgery [5]. Pasalic has introduced a rare case of supratentorial and infratentorial localized angiofibroma who was

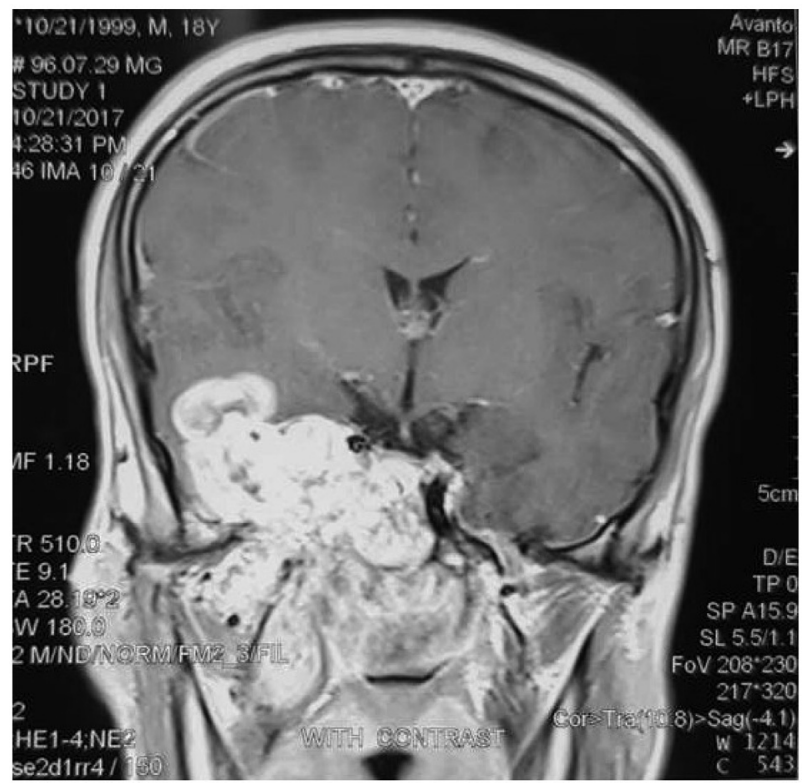

Fig. 3. MRI showed a hypervascular mass with plenty of flow voids signal, due to containing large vessels, in the right nasal cavity with extension to intracranial and infratemporal spaces. initially presented with symptoms of increased intracranial pressure [6].

Celik et al presented four atypical cases of angiofibroma with unusual localization of the tumor like tonsillar bed or inferior concha [4]. Vasconcelos $\mathrm{AC}$ et al reported an atypical angiofibroma with a soft palate lesion [7].

But we did not find any report about intraluminal growth of this tumor. This phenomenon is occasionally seen in glomus jugular and there are some case reports about it [3]. But, it has not been reported in literature.

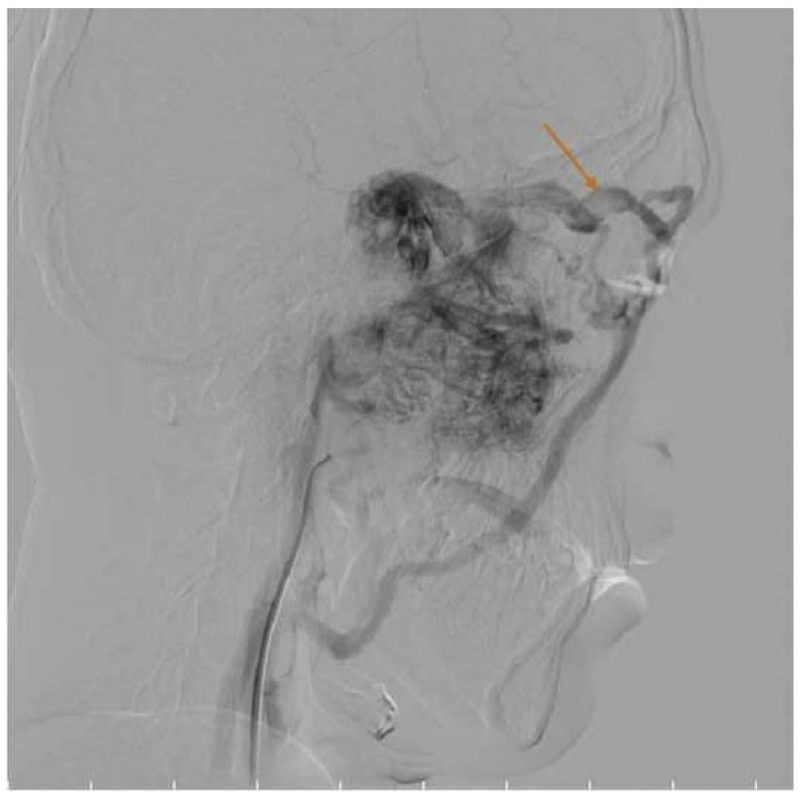

Fig. 4. The angiographic study showed a filling defect in ophthalmic vein according to intraluminal tumor growth. 


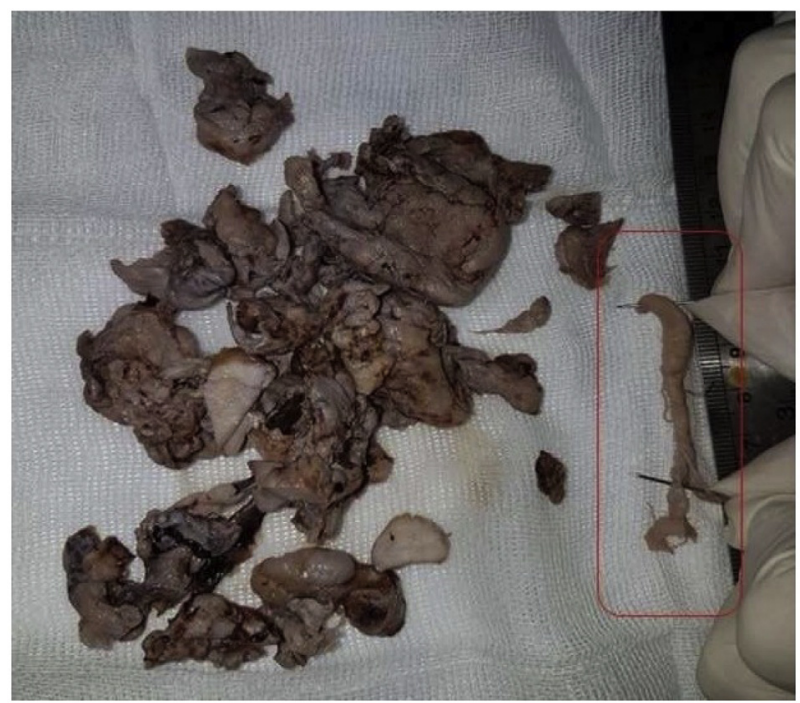

Fig. 5. The excised specimen with an intraluminal extension in to ophthalmic vein (marked in the red box), which was completely excised endoscopically.

The definitive treatment of angiofibroma is surgical excision. Different surgical approaches are used but nowadays endoscopic resection with or without pre-operative embolization is the first choice of treatment. Although the tumors that involve multiple compartments can be challenging to access surgically [8].

We performed an embolization followed by endoscopic resection due to extensive tumor and it was successfully excised via endoscopic surgery. The intraluminal growth of the tumor was also excised as a pedunculated mass separately (Fig. 4).

\section{Conclusion}

Juvenile angiofibroma which is a rare benign tumor with aggressive growth pattern, can invade adjacent critical structures like intracranial or infratemporal fossa. In this manuscript, we present a unique intraluminal growth of the tumor into the ophthalmic vein, which should be keep in mind as one way of its extension.

\section{Conflict of interest statement}

I declare i have no conflict of interest.

\section{Funding}

This research did not receive any specific grant from funding agencies in the public, commercial, or not-for-profit sectors.

\section{References}

[1] Ardehali MM, Samimi Ardestani SH, Yazdani N, Goodarzi H, Bastaninejad S. Endoscopic approach for excision of juvenile nasopharyngeal angiofibroma: complic ations and outcomes. Am J Otolaryngol 2010 Sep-Oct;31(5):343-9.

[2] Szymańska A, Szymański M, Czekajska-Chehab E, SzczerboTrojanowska M. Invasive growth patterns of juvenile nasopharyngeal angiofibroma: radiological imagi ng and clinical implications. Acta Radiol 2014 Jul;55(6):725-31.

[3] Husband AD1, Spedding A, Davis AE. Neck mass caused by an intraluminal jugular paraganglioma. J Laryngol Otol 2000 May;114(5):389-91.

[4] Celik B, Erisen L, Saraydaroglu O, Coskun H. Atypical angiofibromas: a report of four cases. Int J Pediatr Otorhinolaryngol 2005 Mar;69(3):415-21.

[5] Mørkenborg ML, Frendø M, Stavngaard T, Von Buchwald C. Bilateral, independent juvenile nasopharyngeal angiofibroma: case report. J Laryngol Otol 2015 Oct;129(10):1032-5.

[6] Pašalić I, Trninić I, Nemir J, Jednačak H, Žarković K, Mrak G. Rare Infratentorial and Supratentorial Localization of Juvenile Angiofibroma: A Case Report. Pediatr Neurosurg 2016;51(6): $313-7$.

[7] Vasconcelos AC, de Freitas Filho SAJ, de Almeida VL, Caetano RDS, Tramujas J, Paranhos LR. Atypical angiofibroma in a patient with compromised general health: A case report. Ann Med Surg (Lond) 2018 Sep 28;35:133-6.

[8] Liu JK, Husain Q, Kanumuri V, Khan MN, Mendelson ZS, Eloy JA. Endoscopic graduated multiangle, multicorridor resection of juvenile nasopharyngeal angiofibroma: an individualized, tailored, multicorridor skull base approach. J Neurosurg 2016 May;124(5):1328-38. 